\title{
A within-session effect after prolonged training in probability learning by rats ${ }^{1}$
}

CHARLES P. SHIMP, The University of
Utah, Salt Lake City, Utah 84112

Five rats performed in a discrete-trials two-choice probability-learning experiment with a correction procedure. The constant probability of reinforcement for the less frequently reinforced choice $\left(A_{1}\right)$ was $\pi=.3$. Training continued for 12,500 trials-100 per day for 125 days. At asymptote, the relative frequency of $A_{1}$ was .39 on Trial $1, .26$ over Trials $1-4$, but only .06 over Trials 90-100. This within-session effect suggests a new interpretation of the probability-matching vs maximizing controversy.

The discrete-trials probability-learning experiment with a correction procedure has produced inconsistent results with rat Ss. Some studies have found matching (Lauer \& Estes, 1954; Hickson, 1961; Witte, 1961), i.e., the relative frequency of choosing one alternative approximately equaled the relative frequency of reinforcement of that alternative. Other studies, however, have found a tendency to maximize (Uhl, 1963; Calfee, 1968), i.e., the relative frequency of choosing the more frequently reinforced alternative exceeded the corresponding relative frequency of reinforcement. The common explanation of Es who found maximizing for why other Es obtained matching is that training was insufficient to obtain steady-state behavior. This explanation has seemed reasonable since Lauer and Estes, Hickson, and Witte ran their rats for 56, 160 , and 500 trials, respectively, whereas Uhl and Calfee ran their rats for 1,000 and 5,600 trials (for constant $\pi$ ), respectively. An alternative explanation relates to the difference in the number of trials per session. Lauer and Estes, Hickson, and Witte ran their rats for 1 or 4,4 , and 5 trials per session, respectively, whereas Uhl and Calfee ran their rats for 40 , and 80 or

Fig. 1. The top panel shows the learning curves of three rats. $\mathbf{P}\left(\mathbf{A}_{1}\right)$ is the relative frequency of $A_{1}$ in a session of 100 trials. The initial values of $P\left(A_{1}\right)$ approximate $\pi=.3$, but later values deviate towards 0 . The middle panel shows that over initial sessions, there was no within-session effect. The bottom panel shows that by the end of training, a within-session effect had developed such that the relative frequency of $A_{1}$ approximated $\pi$ on early trials but approximate 0 on later trials.
160 trials per session, respectively. Matching has been obtained when the number of trials per session was low, and a tendency to maximize has been obtained when the number of trials per session was relatively high. This difference suggests that both matching and a tendency to maximize may be obtained, but over different portions of a session. That is, a within-session effect might account for the differing results described above.

\section{SUBJECTS}

Five experimentally naive male albino rats of the Sprague-Dawley strain were maintained at $75 \%$ of their free-feeding weights. They were 90 to 155 days oid at the beginning of training.

\section{APPARATUS}

The two-bar experimental chamber was $11 \frac{1 / 2}{1} \times 14 \times 7 \frac{3}{4}$ in. and was placed in a modified picnic icebox. A Plexiglas wall extended from the ceiling to a floor of
$1 / 2$-in. hardware cloth. This wall was mounted midway between the two bars at one end of the chamber and extended to within 2 in. of the foodcup, which rested on the floor and was mounted in the middle of the opposite end of the chamber. White noise masked extraneous sounds and, by means of different intensities, distinguished the intertrial interval from the time when reinforcement (a P. J. Noyes 45-mg food pellet) was set up.

\section{PROCEDURE}

Each session lasted for 100 discrete trials. A trial consisted of the onset of a 90-dB white noise and one or more responses, the last of which was reinforced. The reinforced response started the intertrial interval, which was indicated by a white noise intensity of $45 \mathrm{~dB}$. Every bar press in the intertrial interval reset the 1 -sec intertrial interval timer.

The choice response was defined to be the first response within a trial. A correction procedure allowed any number of unreinforced responses on one bar, in the presence of the $90-\mathrm{dB}$ noise, to precede the reinforced response on the other bar.
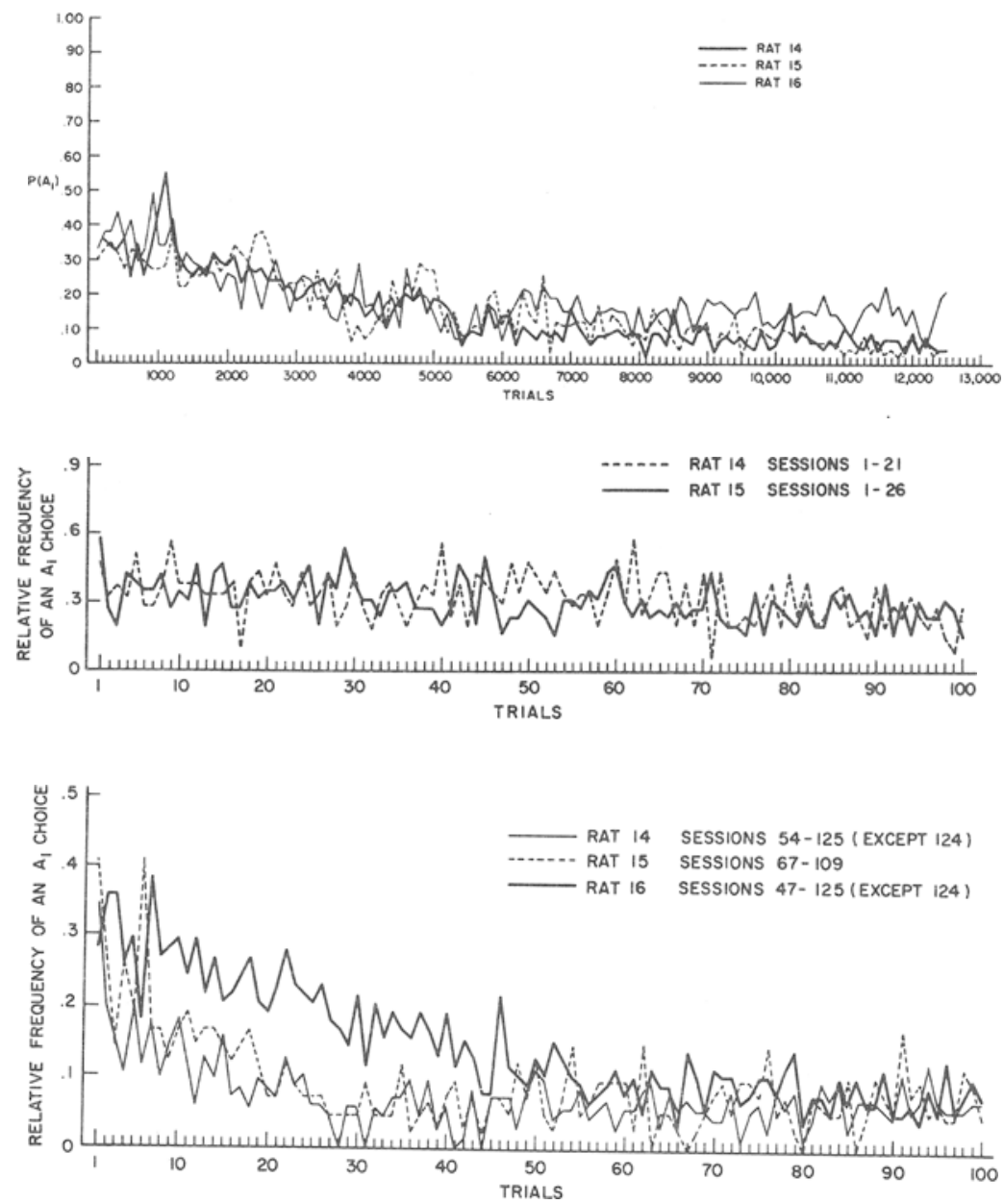
On each trial, reinforcement was available for a response on one of the two bars. The less frequently reinforced choice is denoted by $A_{1}$ and the other choice by $A_{2}$. The relative frequency of $A_{1}$ per session is denoted $P\left(A_{1}\right)$. The constant probability of reinforcement for an $A_{1}$ was $\pi=.3$. The sequence of reinforcements, typically changed each day, was obtained from a table of random numbers and was unrestricted except that the subsequence for Trials 68-100 was the same as for Trials 1-33. Rats 11 and 12 had one sequence and Rats 14, 15, and 16 another.

Every $S$ received magazine and bar training in the experimental chamber. Pretraining was terminated as soon as an animal singly alternated for approximately 10 trials. The number of reinforcements presented during pretraining ranged from 60 to 200 .

\section{RESULTS}

The learning curves of Rats 14,15 , and 16 appear in the top panel of Fig. 1. Over the first several sessions, $P\left(A_{1}\right)$ roughly approximated $\pi$. The learning curves for Rats 14 and 15 were sufficiently stable, over the first 21 and 26 sessions, respcctively, to enable the calculation of the relative frequency of $A_{1}$ for each of the 100 trials. The middle panel of Fig, 1 shows these data. As this panel shows, there was little, if any, within-session effect over these initial sessions: the relative frequency of $\mathbf{A}_{1}$ approximately equaled $\pi$ for each of the: 100 daily trials, for more than 2,000 trials. However, the bottom panel in Fig. 1 shows that a within-session effect had developed by the 50th session. The rate of the within-session change is quite high for Rats 14 and 15 but is more gradual for Rat 16.

Learning curves for Rats 11 and 12 were somewhat steeper than were those for Rats 14,15 , and 16 , shown in the top panel of Fig. 1. Like Rats 14, 15, and 16, the flat within-session curve that was obtained early in training from Rats 11 and 12 changed later to steep curves much like those for Rats 14 and 15 .

Averaging over steady-state behavior of the five rats, the relative frequency of $A_{1}$ on Trial 1 was .39 . Over Trials $1-4$, it was .26. But over Trials 90-100, it was only .06 .

\section{DISCUSSION}

The obtained learning curves are consistent with the view that initial performance by rats in discrete-trials probability-learning experiments using a correction procedure approximates probability matching, but that extended training generates performance more nearly approximating maximizing. However, the obtained within-session effect qualifies this view. The relative frequency of a particular choice on the early trials of terminal sessions approximated the reinforcement probability. Thus, it may well be that the matching obtained by Lauer \& Estes (1954) is a genuine effect and not the result of too little training. However, if such asymptotic matching is found only in nart of a session but disppears over trials after extended training, the linear operator model (Estes, 1964) still requires modification.

\section{REFERENCES}

CALFEE, R. C. Choice behavior during long-term probabilistic reinforcement schedules. Journal of Comparative \& Physiological Psychology, $1968,65,232-237$.

ESTES, W. K. Probability learning. In A. W. Melton (Ed.), Categories of human learning. New York: Academic Press, 1964. Pp. 89-128.

HICKSON, R. H. Response probability in a two-choice learning situation with varying probability of reinforcement. Journal of
Experimental Psychology, 1961, 62, 138-144. LAUER, D. W., \& ESTES, W. K. Observed and predicted terminal distributions of response probability under two conditions of random reinforcement. American Psychologist, 1954, 9, 413. (Abstract)

UHL, C. N. Two-choice probability learning in the rat as a function of incentive, probability of reinforcement, and training procedure. Journal of Experimental Psychology, 1963, $66,443-449$.

WITTE, R. S. Conditioned response probability in a T-maze. Journal of Experimental Psychology, 1961, 62, 439-447.

NOTE

1. This research was performed while the author was at Brown University; it was supported in part by a NSF Graduate Fellowship. The author would like to thank Dr. Richard B. Millward for his many helpful suggestions during the course of this research. The preparation of this manuscript was supported in part by a $\mathrm{MH}$ grant (No. 16928) to the author.

\section{Orienting response of kittens with lesions of the superior colliculus ${ }^{1}$}

THOMAS S. BROWN, Institute for Psychosomatic and Psychiatric Research and Training, Michael Reese Hospital, Chicago, Ill. 60616, and GERSHON BERKSON, Illinois State Pediatric Institute, Chicago, Ill. 60608

Kittens whose superior colliculi had been ablated showed a defect in the eye-fixation response but not in heart-rate or respiration-rate responses.

Dreher, Marchiafava, \& Zemicki (1965) have shown that ablation of the superior colliculus in adult cats reduces eye fixations markedly and also affects head movements, pupillary dilation, and EEG arousal. The purpose of this experiment was to examine the effects of lesions of the superior colliculus on eye fixation, heart rate, and respiration rate in the kitten. METHOD

The Ss were 16 mother-reared kittens, approximately 60 days of age at the time of testing. Five of the Ss had received bilateral ablation of the superior colliculus at 50 days of age. These animals were the $\mathrm{SC}$ group, and the remaining $11 \mathrm{Ss}$ were the control group.

The SC group received one-stage bilateral aspirations of the superior colliculus under Nembutal anesthesia $(30 \mathrm{mg} / \mathrm{kg})$. A bilateral crainotomy was performed over the posterolateral area of the skull, the posterior boundary of the bone removal being the tentorium. When the bone removal was judged sufficient, the dura was opened and the head of the animal was then rotated forward so that the animal faced downwards. The cerebral hemispheres were then gently retracted forward away from the bony tentorium by inserting strips of cottonoid between the tentorium and the posterior aspect of the hemisphere. The colliculi were thus visualized and aspirated. The wound was closed in anatomical layers, and the animal was treated with antibiotics.

Apparatus and Procedure

The animal was restrained in a holder that was placed in a $2 \times 2 \times 2 \mathrm{ft}$. sound-deadened cubicle from which external light was excluded. The animal faced the front wall of the cubicle, which served as the door and also as a stimulus panel. At S's eye level in the center of the door was a 0.25 -in.-diam peephole through which the animal could be observed from outside the cubicle. On either side of the peephole was a $3.5 \times 3.75$ in. rectangular stimulus panel whose center was $6.50 \mathrm{in}$. from the peephole. In these experiments, the panel to S's right was covered with opaque material. The other panel was covered with frosted glass which could be lit from behind two 6-W bulbs. In front of this panel was a 2.25-in.-diam disk which could rotate, and when it did so, an $89-\mathrm{dB}$ 